The Astrophysical Journal, Vol. 149, August 1967

\title{
THE EINSTEIN REDSHIFT IN WHITE DWARFS*
}

\author{
Jesse L. Greenstein and Virginia L. Trimble \\ Mount Wilson and Palomar Observatories, Carnegie Institution of Washington, \\ California Institute of Technology \\ Received February 1, 1967
}

\begin{abstract}
Low-dispersion radial velocities of 53 white dwarfs have been measured on Palomar spectrograms. Table 1 contains the type, velocity, space-motion components, photometrically deduced temperature and radius, for each star. Table 4 contains 39 additional radial velocities of very low weight. A few members of wide binary systems and 6 white dwarfs in the Hyades provide direct measures of the Einstein gravitational redshift, with a mean value of $+51 \mathrm{~km} / \mathrm{sec}$. Omitting the very-high-velocity star LP9-231, there are $37 \mathrm{DA}$ stars, with a mean $\mathrm{K}$-term (expansion velocity) of $+65.6 \mathrm{~km} / \mathrm{sec}$. If the Hyades stars are omitted, the mean $K$ term is $+62.5 \mathrm{~km} / \mathrm{sec}$ A number of white dwarfs are members of the high-velocity population. Systematic wavelength shifts of He I lines in DB stars make their velocities more negative than those of DA stars; similar negative shifts may exist for metallic lines.

The temperature scale is obtained from colors and, combined with luminosities, gives radii. The broad distribution of radii and redshifts is shown in Figure 2, and median values are derived. The median radial velocity for $37 \mathrm{DA}$ stars is $+58 \mathrm{~km} / \mathrm{sec}$, and the median radius $0.0107 R \odot$; the redshift and radius give a mass of $0.98 M \odot$. However, this value is almost certainly too high, if we expect accordance with the theoretical mass-radius relation. The theoretical $M-R$ relation of a zero-temperature degenerate star predicts a redshift, for given mass, for various compositions. Two corrections could bring the theoretically expected redshifts into agreement with the observations. Either a systematic change in luminosity, $\Delta M_{v}$ of $+0.25 \mathrm{mag}$, or a reciprocal temperature change of $\Delta \theta=-0.03$, reduces the median radius to $0.0093 R \odot$. The mass derived from the redshift is then $0.86 M \odot$. These values are in accordance with the Hamada-Salpeter mass-radius relation, if the composition in the interior is pure helium. A carbon or magnesium interior also gives a radius not too different from the colorimetric radius. An iron core gives a mass of $0.73 M \odot$, but a radius of $0.008 R \odot$, sufficiently smaller to require substantial changes in the temperature scale. The mass now derived from the radial velocities is higher than that previously found from radii only and closer to the Chandrasekhar limit
\end{abstract}

\section{INTRODUCTION}

Data concerning the radial velocities and the gravitational redshift of degenerate stars have been severely limited by difficulty of observation and of measurement of the few broad lines. Except for 40 Eri B (Popper 1954) and the difficult, incomplete measurements of $a \mathrm{CMa} \mathrm{B}$ by Oke and Greenstein, no appreciable number of reliable velocities exist. We provide a first catalogue of a number of radial velocities, still inaccurate, but usable for statistical purposes.

Data concerning spectra, colors, and space motions have been provided in recent surveys by Eggen and Greenstein (1965a, 1967; Papers I, II, and III, respectively). During the twelve years of Palomar observations by one of us (JLG), many attempts have been made to measure the velocities. In this investigation the difficulties, we hope, have been circumvented. We present fifty-three velocities which we view as reliable, and another thirty-nine which have little value except as a statistical record. Spectra of about 210 white dwarfs have now been observed, and some 350 actual spectrograms were available. The low yield of usable velocities clearly demonstrates the difficulty of the problem.

\section{TECHNIQUES}

At the Palomar prime-focus spectrograph, $190 \AA / \mathrm{mm}$ spectra of a fifteenth-magnitude star, widened by $0.3 \mathrm{~mm}$, are obtained in 1 hour. The normal slit-width is 0 ". 8 , i.e., slight-

* This research was supported in part by the U S. Air Force under contract AF 49(638)-1323, monitored by the Air Force Office of Scientific Research of the Office of Aerospace Research. 
ly smaller than the stellar image in average good seeing. The radial-velocity shift is 11 $\mathrm{km} / \mathrm{sec}$ per micron on the plate; the slit-width corresponds to about $350 \mathrm{~km} / \mathrm{sec}$. Therefore, both mechanical stability of the spectrograph, and careful, symmetrical guiding of the star on the slit are prime necessities. For this reason we should not be surprised by "huge" errors; differences between velocities given by two plates of the same star may on occasion exceed $100 \mathrm{~km} / \mathrm{sec}$.

In the most common type of blue white dwarf, DA, the hydrogen lines have halfwidths $w_{05}$ from 30 to $50 \AA$; the DAs have $w_{05}$ of $20 \AA$. At best the equivalent velocity half-width is $1500 \mathrm{~km} / \mathrm{sec}$, and only from three to six Balmer lines are ever measurable. (We have no plates of $\mathrm{Ha}$.) Usually $\mathrm{H} \beta, \mathrm{H} \gamma, \mathrm{H} \delta, \mathrm{H} \epsilon$, and $\mathrm{H} \zeta$ are used; $\mathrm{H} \beta$, because of its location on the decreasing density slope at the end of the spectrum, is often systematically in error and must be discarded. Even $\mathrm{H} \gamma$ is on a sloping background (Weidemann 1963). We cannot average errors of measurement from many lines and a plate with a single sharp defect located near the center of one broad line will yield a very discordant velocity.

First measurements with a conventional microscope and cross-hair machine were disappointing. One psychological difficulty was that the black emission line of the comparison $(\mathrm{H}, \mathrm{He})$ distracted the eye during its attempt to locate the center of the broad, gray absorption line. The Grant oscilloscope measuring machine (Tomkins-Fred principle) displays rapidly repeated photometric scans on the oscilloscope screen, showing a line simultaneously in direct and reverse directions. The setting consists in matching the direct and reverse scans - either of the core of the line or of some midpoint in the wings. Only part of the wings, a total of about $30 \AA$, was visible on the screen. Every usable spectrum was inspected and measured between two and four times, in one homogeneous, continued series by one of us (VT). It is, of course, impossible to use normal velocity standards, since none exists with this type of line profile. Even rapidly rotating B stars with $v \sin i$ of $300 \mathrm{~km} / \mathrm{sec}$ have $w_{0.5}$ of only $8 \AA$; they would also be far too bright to be observed in a manner homogeneous with the white dwarfs. Therefore, the internal consistency provides our only guide, at present, to the questions of possible systematic error of the velocity system. It is hoped that other observatories will attempt an independent determination.

The measuring machine, essentially, sweeps a slit across the line in about a millisecond, and then repeats, presenting the next scan in the reversed direction on the oscilloscope. One plate grain is traversed in a few microseconds. Non-uniformity of illumination, in space and time, inexact reversibility of charging and discharging circuits, and non-linearity of the horizontal-sweep time base may produce a systematic background drift of opposite sign between forward and backward sweeps. The extreme line wings would have opposite asymmetries. After study, it was found necessary to remeasure each spectrogram after rotation by $180^{\circ}$, to eliminate possible systematic instrumental effects. Density gradients in the background continuum, however, cannot be eliminated; $\mathrm{H} \beta$ is therefore given low weight or omitted. Each plate was measured one to three times in the direct sense, to test quality, and once in the reverse sense. The direct measures were averaged and then combined with the reverse measure with equal weight. It was found that measures by VT made in the sense red to blue gave velocities $20 \mathrm{~km} / \mathrm{sec}$ larger than those in the sense blue to red. The systematic, instrumental measuring error in one direction, therefore, was $10 \mathrm{~km} / \mathrm{sec}$, or $1 \mu$ on the plate. This is surprisingly small when the half-width of $\mathrm{H} \gamma$ corresponds to $200 \mu$. Different experienced measurers develop significantly different systematic errors; for example, JLG - VT $\approx$ $+20 \pm 7 \mathrm{~km} / \mathrm{sec}$ in the direct, and $-40 \pm 5$ in the reverse measures. The rotation of the spectra, and averaging direct and reverse, results in cancellation of these systematic effects within the probable error of their determination. Such effects arise from changes, between observers, of the emphasis given to setting on the line core as against part way $i$ to the wings. 
The lines, as measured by VT, were weighted equally, except for $\mathrm{H} \beta$, which, when accordant, was given half-weight, but otherwise zero weight. The comparison lines were of $\mathrm{H}$ and $\mathrm{He}$; sometimes $\mathrm{Ar}$ II and $\mathrm{Ne}$ II were added. The repeatability of the measurements was very high, averaging between 1 and $2 \mu$. The source of the true uncertainty, which is about $5 \mu$, is a decision-making process as to the proper balance between setting on the core, the nearer parts of the wing, or on a grain irregularity. Velocities derived from the VT measures in a given direction show an average deviation between two plates of a star equal to $\pm 23 \mathrm{~km} / \mathrm{sec}$. Then the external mean error of a measure in one direction is $\pm 18 \mathrm{~km} / \mathrm{sec}$. Since all plates are measured direct and reverse, the indicated mean error of a good velocity is $\pm 13 \mathrm{~km} / \mathrm{sec}$. The internal mean error from the interagreement of the lines, was near $\pm 20 \mathrm{~km} / \mathrm{sec}$; the accordance, externally, is slightly better than is expected from the internal errors, in part because we reject externally discordant pairs of spectra.

\section{PRESENTATION OF DATA}

In Table 1 we tabulate those velocities which we view as reliable. In column I they are listed first by EG number; the second line gives the number of plates measured and the spectral type; if the type is not given, it is DA; line 3 gives another identifying name. In column II, the observed radial velocity, $\rho_{i}$, is given first; the second line is the radial velocity corrected for normal solar motion $(\rho-V \odot \cos \lambda)$, and the last $\rho-K^{\prime}$, the velocity corrected for the mean redshift. Here $K^{\prime}=\left\langle\rho_{i}\right\rangle$ is the mean expansion velocity of this entire group of fifty-three white dwarfs. We assume $\Sigma \rho_{i}=\Sigma k_{i}$, where $k_{i}$ is the gravitational redshift of each star. The third entry in column II is given in parentheses where the true radial velocity is otherwise known, e.g., for the white-dwarf members of the Hyades and of visual binary systems and is $k_{i}$ itself. These individually determined $k_{i}$ are summarized in Table 2 . The quantity $\rho-K$ (or $\rho-k_{i}$ ) is used as the true radial motion to compute the space-motion vectors $U, V$, and $W$ in column III, for the brighter possible luminosity law (Eggen and Greenstein 1965a), and in column IV for the fainter possible luminosity. Only one set of $U V W$ is given when the luminosity is known unambiguously. Column $\mathrm{V}$ contains $-\log R / R \odot$ and $\theta_{e}=5040 / T_{e}$ derived from luminosity and $U B V$ colors (see below). Low-dispersion radial velocities for red companions listed in Table 2 are in parentheses; resultant redshifts are in parentheses in column $I$ of Table 1.

\section{SPACE MOTIONS}

The goal of this program was the determination of radial velocities, but as a by-product we have data on motions, that is, forty-two values of $U, V, W$; there are six Hyades stars, which we will count as one object; we must omit LP9-231 because of excessively high velocity; and sixteen have ambiguous luminosity. The material is too small for a new determination of solar motion, since the group contains some high-velocity stars. We have eliminated the redshift, statistically, and also the solar radial velocity with respect to the normal apex of low-velocity stars, which is $U=-10, V=+14, W=$ $+7 \mathrm{~km} / \mathrm{sec}$. This apex probably differs from that of the white dwarfs by about $30^{\circ}$ and the second-order effects would appear were we to recompute $\Sigma k_{i}{ }^{\prime}$, the redshifts, from $\left\langle\rho-V \odot^{\prime} \cos \lambda^{\prime}\right\rangle$, where primes refer to the corrected apex. The corrected redshift would then change slightly the star's true radial velocity and therefore the amount subtracted from the old $U_{0}, V_{0}, W_{0}$ values given in Papers I and III. Since Eggen is planning a full discussion of the space motions of white dwarfs, only a very rough treatment will be given here. For twenty-eight stars assumed to lie on the upper sequence, we find $\langle U\rangle=+15 \pm 9,\langle V\rangle=-26 \pm 5$, and $\langle W\rangle=-13 \pm 5 \mathrm{~km} / \mathrm{sec}$. For twenty-one stars (lower sequence), these values are $+5 \pm 10,-22 \pm 6$, and $-21 \pm 5 \mathrm{~km} / \mathrm{sec}$, respectively. The average deviations are estimated as $\langle|U|\rangle= \pm 45,\langle|V|\rangle= \pm 22$ $\langle|W|\rangle= \pm 23 \mathrm{~km} / \mathrm{sec}$. Pavlovskaya (1956) determined a solar motion with respect to 
TABLE 1

RADIAL VELOCITIES, SPACE MOTIONS, RADII AND TEMPERATURES

\begin{tabular}{|c|c|c|c|c|c|c|c|c|c|}
\hline$I$ & II & III & IV & V & $I$ & II & III & IV & $\mathrm{V}$ \\
\hline \begin{tabular}{|l}
3 \\
$4 \mathrm{DB}$ \\
$\mathrm{F} 4$
\end{tabular} & $\begin{array}{l}+69 \\
+\quad 69 \\
+19\end{array}$ & $\begin{array}{l}+16 \\
+\quad 7 \\
-14\end{array}$ & $\overline{--}$ & - & $\begin{array}{l}62 \\
\text { 2DA, Fs } \\
\mathrm{L} 532-81\end{array}$ & $\begin{array}{l}+24 \\
+\quad 8 \\
-\quad 27\end{array}$ & & $\begin{array}{l}+70 \\
+47 \\
-19\end{array}$ & \\
\hline $\begin{array}{l}4 \\
1 \\
\text { G1-7 }\end{array}$ & $\begin{array}{l}+108 \\
+102 \\
+57\end{array}$ & $\begin{array}{l}+114 \\
-\quad 37 \\
-56\end{array}$ & $\begin{array}{l}+74 \\
-12 \\
-53\end{array}$ & $\begin{array}{r}-1.67 \\
-1.97 \\
0.55\end{array}$ & $\begin{array}{l}64 \\
1 \\
\text { G116-16 }\end{array}$ & $\begin{array}{c}+74 \\
+72 \\
(+131)\end{array}$ & & $\begin{array}{l}-44 \\
-58 \\
-34\end{array}$ & $\begin{array}{c}-1.75 \\
\left.(-57)^{2}\right) \\
0.55\end{array}$ \\
\hline $\begin{array}{l}167 \\
1 \\
6132-12\end{array}$ & $\begin{array}{l}+102 \\
+104 \\
+51\end{array}$ & & & & $\begin{array}{l}71 \\
1 D O \\
\text { F34 }\end{array}$ & $\begin{array}{l}+17 \\
+19 \\
-34\end{array}$ & & $\begin{array}{l}-21 \\
-3 \\
-13\end{array}$ & $\begin{array}{r}-2.14 \\
0.10\end{array}$ \\
\hline $\begin{array}{lll}5 & & \\
9 \mathrm{DG} & \\
\mathrm{V} & \text { Ma } & 2\end{array}$ & $\begin{array}{l}+54 \\
+54 \\
+\quad 3\end{array}$ & & $\begin{array}{l}+3 \\
-83 \\
-70\end{array}$ & & $\begin{array}{l}73 \\
2 \\
T 547\end{array}$ & $\begin{array}{l}+41 \\
+42 \\
-10\end{array}$ & & & $\begin{array}{r}-1.76 \\
-2.02 \\
0.52\end{array}$ \\
\hline $\begin{array}{l}7 \\
1 \\
61-45\end{array}$ & $\begin{array}{l}+61 \\
+58 \\
+10\end{array}$ & $\begin{array}{l}+61 \\
+20 \\
+\quad 22\end{array}$ & $\begin{array}{l}+36 \\
+13 \\
+9\end{array}$ & $\begin{array}{r}-1.71 \\
-1.99 \\
0.52\end{array}$ & $\begin{array}{l}75 \\
1 \\
\text { LB 253 }\end{array}$ & $\begin{array}{r}+58 \\
+\quad 64 \\
+\quad 7\end{array}$ & $\begin{array}{l}+30 \\
-\quad 36 \\
+\quad 7\end{array}$ & $\begin{array}{l}+18 \\
-18 \\
+\quad 6\end{array}$ & $\begin{array}{r}-1.74 \\
-2.00 \\
0.39\end{array}$ \\
\hline $\begin{array}{l}168 \\
1 \\
\text { G74-7 }\end{array}$ & $\begin{array}{l}+29 \\
+29 \\
-22\end{array}$ & & & & $\begin{array}{l}79 \\
2 \mathrm{DF} \\
\mathrm{R} 627\end{array}$ & $\begin{array}{l}+62 \\
+62 \\
+11\end{array}$ & & $\begin{array}{l}+49 \\
-22 \\
-37\end{array}$ & $\begin{array}{r}-2.10 \\
0.61\end{array}$ \\
\hline $\begin{array}{l}19 \\
2 \\
\text { F22 }\end{array}$ & $\begin{array}{l}+30 \\
+23 \\
-21\end{array}$ & $\begin{array}{l}-4 \\
-\quad 17 \\
+18\end{array}$ & & $\begin{array}{r}-1.78 \\
0.23\end{array}$ & $\begin{array}{l}80 \\
2 \\
\text { F43 }\end{array}$ & $\begin{array}{l}+110 \\
+114 \\
+\quad 60\end{array}$ & $\begin{array}{l}+38 \\
-24 \\
+50\end{array}$ & & $\begin{array}{r}-1.81 \\
0.28\end{array}$ \\
\hline $\begin{array}{l}23 \\
2 \\
G 37-44\end{array}$ & $\begin{array}{l}+90 \\
+83 \\
+39\end{array}$ & $\begin{array}{l}+89 \\
-133 \\
-\quad 20\end{array}$ & $\begin{array}{l}+60 \\
-54 \\
-16\end{array}$ & $\begin{array}{r}-1.68 \\
-2.02 \\
0.54\end{array}$ & $\begin{array}{l}92 \\
1 \\
\text { G61-17 }\end{array}$ & $\begin{array}{l}+\quad 2 \\
+\quad 6 \\
-50\end{array}$ & $\begin{array}{c}+164 \\
0 \\
-\quad 34\end{array}$ & & $\begin{array}{r}-1.66 \\
0.37\end{array}$ \\
\hline $\begin{array}{l}26 \\
1 \\
\mathrm{HZ} 4\end{array}$ & $\begin{array}{c}+105 \\
+92 \\
(+69)\end{array}$ & $\begin{array}{l}+41 \\
-\quad 20 \\
-\quad 2\end{array}$ & & $\begin{array}{r}-1.73 \\
0.52\end{array}$ & $\begin{array}{l}189 \\
1 \\
\text { GD163 }\end{array}$ & $\begin{array}{l}+23 \\
+36 \\
-28\end{array}$ & $\begin{array}{l}-17 \\
-30 \\
-24\end{array}$ & $\begin{array}{l}-12 \\
-25 \\
-25\end{array}$ & $\begin{array}{r}-1.95 \\
-2.07 \\
0.38\end{array}$ \\
\hline \begin{tabular}{|l}
28 \\
2 \\
$\mathrm{LB} 1240$
\end{tabular} & $\begin{array}{c}+88 \\
+78 \\
(+52)\end{array}$ & & $\begin{array}{l}+35 \\
-17 \\
-16\end{array}$ & $\begin{array}{r}-1.89 \\
0.51\end{array}$ & $\begin{array}{l}107 \\
2 \\
\text { F93 }\end{array}$ & $\begin{array}{l}+97 \\
+108 \\
+46\end{array}$ & & & $\begin{array}{r}-1.92 \\
0.20\end{array}$ \\
\hline $\begin{array}{l}30 \\
1 \\
\mathrm{HZ} 10\end{array}$ & $\begin{array}{c}+80 \\
+70 \\
(+43)\end{array}$ & $\begin{array}{l}+35 \\
-16 \\
-15\end{array}$ & & $\begin{array}{r}-1.70 \\
0.52\end{array}$ & $\begin{array}{l}108 \\
1 \\
\text { T197 }\end{array}$ & $\begin{array}{l}+51 \\
+65 \\
0\end{array}$ & & & $\begin{array}{r}-2.03 \\
0.27\end{array}$ \\
\hline $\begin{array}{l}31 \\
2 \\
\mathrm{HZ} 2\end{array}$ & $\begin{array}{c}+64 \\
+51 \\
(+27)\end{array}$ & $\begin{array}{l}+32 \\
-18 \\
-20\end{array}$ & & $\begin{array}{r}-1.75 \\
0.36\end{array}$ & $\begin{array}{l}113 \\
1 \\
+10_{3129 B}\end{array}$ & $\begin{array}{c}+48 \\
+64 \\
(+52)\end{array}$ & & & $\left.\begin{array}{c}-2.05 \\
(-4.12\end{array}\right)$ \\
\hline $\begin{array}{l}170 \\
\text { IDF } \\
\text { G82-23 }\end{array}$ & $\begin{array}{l}+2 \\
-15 \\
(-35)\end{array}$ & & & $(+37)$ & $\begin{array}{l}116 \\
1 \\
c 2\end{array}$ & $\begin{array}{l}+27 \\
+45 \\
-\quad 24\end{array}$ & & & $\begin{array}{r}-1.70 \\
-1.98 \\
0.49\end{array}$ \\
\hline $\begin{array}{l}39 \\
1 \\
\mathrm{HZ7}\end{array}$ & $\begin{array}{c}+118 \\
+104 \\
(+79)\end{array}$ & $\begin{array}{l}+46 \\
-13 \\
-\quad 3\end{array}$ & & $\begin{array}{r}-1.83 \\
0.37\end{array}$ & $\begin{array}{l}119 \\
\text { 2DFp } \\
\text { R640 }\end{array}$ & $\begin{array}{l}+24 \\
+42 \\
-28\end{array}$ & $\begin{array}{l}+141 \\
-\quad 8 \\
+\quad 43\end{array}$ & $\begin{array}{l}+66 \\
-13 \\
+7\end{array}$ & \\
\hline $\begin{array}{l}42 \\
2 \\
\text { HZ14 }\end{array}$ & $\begin{array}{c}+105 \\
+91 \\
(+65)\end{array}$ & $\begin{array}{c}+42 \\
-13 \\
0\end{array}$ & & $\begin{array}{r}-1.89 \\
0.25\end{array}$ & $\begin{array}{l}197 \\
1 \\
\text { G169-34 }\end{array}$ & $\begin{array}{l}+61 \\
+80 \\
+10\end{array}$ & $\begin{array}{l}-87 \\
-\quad 55 \\
-\quad 23\end{array}$ & $\begin{array}{l}-41 \\
-22 \\
-7\end{array}$ & $\begin{array}{r}-1.65 \\
-1.99 \\
0.58\end{array}$ \\
\hline $\begin{array}{l}171 \\
1 \\
\text { G89-10 }\end{array}$ & $\begin{array}{l}+130 \\
+115 \\
+78\end{array}$ & & & & $\begin{array}{l}199 \\
2 \\
L P 9-231\end{array}$ & $\begin{array}{l}-154 \\
-145 \\
-205\end{array}$ & $\begin{array}{l}+286 \\
-472 \\
+\quad 53\end{array}$ & $\begin{array}{l}+180 \\
-269 \\
-\quad 22\end{array}$ & $\begin{array}{c}(-1.54) \\
-1.92 \\
0.71\end{array}$ \\
\hline $\begin{array}{l}54 \\
1 \mathrm{DF} \\
\mathrm{L} 745-46 \mathrm{~A}\end{array}$ & $\begin{array}{l}+11 \\
-\quad 7 \\
-40\end{array}$ & & $\begin{array}{l}-56 \\
+11 \\
+27\end{array}$ & $\begin{array}{r}-2.06 \\
0.60\end{array}$ & $\begin{array}{l}125 \\
1 \\
R 137\end{array}$ & $\begin{array}{l}+67 \\
+84 \\
+16\end{array}$ & $\begin{array}{l}-47 \\
-45 \\
+16\end{array}$ & $\begin{array}{l}-29 \\
-\quad 15 \\
+\quad 8\end{array}$ & $\begin{array}{r}-1.71 \\
-2.03 \\
0.48\end{array}$ \\
\hline $\begin{array}{l}57 \\
1 \\
1 \\
L 817-13\end{array}$ & $\begin{array}{l}+56 \\
+\quad 38 \\
+\quad 5\end{array}$ & $\begin{array}{l}-17 \\
-24 \\
-41\end{array}$ & $\begin{array}{l}-7 \\
-14 \\
-20\end{array}$ & $\begin{array}{r}-1.74 \\
-2.00 \\
0.39\end{array}$ & $\begin{array}{l}126 \\
1 \\
\text { G21-16 }\end{array}$ & $\begin{array}{l}0 \\
+\quad 17 \\
-\quad 51\end{array}$ & $\begin{array}{l}+19 \\
-17 \\
-\quad 34\end{array}$ & $\begin{array}{l}+33 \\
-48 \\
-17\end{array}$ & $\begin{array}{r}-1.63 \\
-1.95 \\
0.58\end{array}$ \\
\hline $\begin{array}{l}58 \\
2 \mathrm{DA}, \mathrm{F} \\
\mathrm{G} 111-71\end{array}$ & $\begin{array}{l}-12 \\
-14 \\
-63\end{array}$ & & $\begin{array}{l}-25 \\
-43 \\
-82\end{array}$ & $\begin{array}{r}-1.97 \\
0.62\end{array}$ & $\begin{array}{l}128 \\
1 \\
\text { G141-54 }\end{array}$ & $\begin{array}{l}-12 \\
+\quad 6 \\
-63\end{array}$ & & & $\begin{array}{r}-1.67 \\
-1.99 \\
0.55\end{array}$ \\
\hline
\end{tabular}

286 
TABLE 1 (CONT'D)

\begin{tabular}{|c|c|c|c|c|c|c|c|c|c|}
\hline$I$ & II & III & IV & $\mathrm{V}$ & $I$ & II & III & IV & $\mathrm{V}$ \\
\hline $\begin{array}{l}130 \\
2 \\
\text { G141B 2B }\end{array}$ & $\begin{array}{l}+65 \\
+83 \\
+14\end{array}$ & $\begin{array}{l}-20 \\
+\quad 1 \\
-\quad 8\end{array}$ & & $\begin{array}{r}-1.73 \\
0.52\end{array}$ & $\begin{array}{l}149 \\
1 D B \\
\text { L930-80 }\end{array}$ & $\begin{array}{l}+20 \\
+28 \\
-31\end{array}$ & $\begin{array}{l}+48 \\
-\quad 49 \\
-\quad 29\end{array}$ & & \\
\hline $\begin{array}{l}201 \\
1 \\
\text { GD } 219\end{array}$ & $\begin{array}{l}+35 \\
+53 \\
-16\end{array}$ & $\begin{array}{l}+3 \\
-\quad 18 \\
-\quad 5\end{array}$ & $\begin{array}{l}+7 \\
-15 \\
-2\end{array}$ & $\begin{array}{r}-1.67 \\
-1.97 \\
0.48\end{array}$ & $\begin{array}{l}151 \\
1 \\
\text { G93-53 }\end{array}$ & $\begin{array}{l}+113 \\
+122 \\
+\quad 62\end{array}$ & $\begin{array}{l}-51 \\
0 \\
-\quad 63\end{array}$ & $\begin{array}{l}-35 \\
+25 \\
-49\end{array}$ & $\begin{array}{r}-1.61 \\
-2.03 \\
0.62\end{array}$ \\
\hline $\begin{array}{l}133 \\
2 \mathrm{DB} \\
\mathrm{L} 1573-31\end{array}$ & $\begin{array}{l}+40 \\
+\quad 6 \\
-\quad 11\end{array}$ & $\begin{array}{l}-40 \\
-\quad 20 \\
-\quad 34\end{array}$ & & & $\begin{array}{l}153 \\
\text { IDB } \\
\text { LDS785A }\end{array}$ & $\begin{array}{l}+44 \\
+\quad 46 \\
-\quad 7\end{array}$ & $\begin{array}{l}+54 \\
-17 \\
-\quad 24\end{array}$ & & \\
\hline $\begin{array}{l}134 \\
1 \\
G 142-50\end{array}$ & $\begin{array}{l}+77 \\
+94 \\
+\quad 26\end{array}$ & $\begin{array}{l}-51 \\
-\quad 9 \\
-\quad 27\end{array}$ & $\begin{array}{l}-37 \\
+\quad 2 \\
-17\end{array}$ & $\begin{array}{r}-1.80 \\
-2.02 \\
0.34\end{array}$ & $\begin{array}{l}154 \\
3 D A, F \\
G 28-13\end{array}$ & $\begin{array}{l}+85 \\
+91 \\
+34\end{array}$ & $\begin{array}{l}-128 \\
-\quad 60 \\
-50\end{array}$ & $\begin{array}{l}-52 \\
+\quad 4 \\
-36\end{array}$ & $\begin{array}{r}-1.63 \\
-1.97 \\
0.60\end{array}$ \\
\hline $\begin{array}{l}139 \\
1 \\
W 1346\end{array}$ & $\begin{array}{l}+38 \\
+55 \\
-13\end{array}$ & $\begin{array}{l}+47 \\
-\quad 29 \\
+\quad 2\end{array}$ & & $\begin{array}{r}-1.76 \\
0.35\end{array}$ & $\begin{array}{l}157 \\
1 \\
\text { F108 }\end{array}$ & $\begin{array}{l}+40 \\
+44 \\
-11\end{array}$ & $\begin{array}{l}-6 \\
-\quad 5 \\
+\quad 12\end{array}$ & & $\begin{array}{r}-2.09 \\
0.14\end{array}$ \\
\hline $\begin{array}{l}144 \\
1 \\
+73^{\circ} 8031\end{array}$ & $\begin{array}{l}+8 \\
+20 \\
-44\end{array}$ & $\begin{array}{l}-30 \\
-\quad 25 \\
-39\end{array}$ & $\begin{array}{l}-22 \\
-32 \\
-26\end{array}$ & $\begin{array}{r}-1.75 \\
-2.03 \\
0.43\end{array}$ & $\begin{array}{l}160 \\
1 \\
\text { C3 }\end{array}$ & $\begin{array}{l}+88 \\
+96 \\
+37\end{array}$ & & & $\begin{array}{r}-1.72 \\
-2.04 \\
0.44\end{array}$ \\
\hline $\begin{array}{l}145 \\
\text { 2DB } \\
\text { LDS749B }\end{array}$ & $\begin{array}{l}-81 \\
-70 \\
(-3)\end{array}$ & & $\begin{array}{l}+94 \\
-55 \\
-\quad 3\end{array}$ & $(-78)$ & $\begin{array}{l}162 \\
1 \\
I 1512-34 B\end{array}$ & $\begin{array}{l}+17 \\
+\quad 23 \\
-\quad 34\end{array}$ & & $\begin{array}{l}-21 \\
-24 \\
+17\end{array}$ & $\begin{array}{r}-1.91 \\
0.52\end{array}$ \\
\hline $\begin{array}{l}146 \\
2 \mathrm{DB} \\
\mathrm{I} 1002-62\end{array}$ & $\begin{array}{l}+40 \\
+50 \\
-11\end{array}$ & $\begin{array}{l}+20 \\
-\quad 8 \\
-\quad 9\end{array}$ & & & & & & & \\
\hline
\end{tabular}

From equation (2) a method for plausible estimates of the minimum gravitational redshift was outlined (end of section IV). These make $W$ an extremal of $\pm 50 \mathrm{~km} / \mathrm{sec}$. The results are for DB stars (EG 3, 149, 153) +2, +20, +20; for DA (EG 4, 80, 151) $+57,+50,+53$, and miscellaneous (EG 5, 119,154$),+74,+43,+35 \mathrm{~km} / \mathrm{sec}$. The method fails if the stars have intrinsically high velocity. The gross mean is $\Delta \mathrm{k}_{i}(\mathrm{~min})=+39 \mathrm{~km} / \mathrm{sec}$. 
the white dwarfs, which is listed by Delhaye (1966) as $-6,+37,+8 \mathrm{~km} / \mathrm{sec}$, in good agreement with our values, after the required change of sign. Pavlovskaya's estimate of the dispersion in each component was $\pm 50, \pm 33, \pm 25 \mathrm{~km} / \mathrm{sec}$, also in agreement with these results.

Since the white dwarfs are not very high-velocity objects, estimates of the minimum gravitational shift can sometimes be obtained from the $W$ velocity, when the geometry is favorable. Let $\rho_{i}$ be the observed and $\rho_{i}(0)$ the true radial velocity of star $i$, and $k_{i}$ its gravitational shift. The $W$ velocity is derived from the values based on proper motions only (which in this paper we will denote as $W_{0}$ ) given by Eggen and Greenstein in Papers I and III:

$$
W=W_{0}+\frac{d W}{d \rho} \rho_{i}(0)=W_{0}+\frac{d W}{d \rho}\left(\rho_{i}-k_{i}\right) .
$$

TABLE 2

Directly Measured Gravitational Shifts (KM/Sec)

\begin{tabular}{|c|c|c|c|c|c|}
\hline \multicolumn{3}{|c|}{ Hyades } & \multicolumn{3}{|c|}{ Hyades Group } \\
\hline EG & $n$ & $k_{i}$ & EG & $n$ & $k_{i}$ \\
\hline \multirow{3}{*}{$\begin{array}{l}26 \\
28 \\
30 \\
31 \\
39 \\
42 . \\
\text { Weighted } \\
\text { mean. }\end{array}$} & \multirow{3}{*}{$\begin{array}{l}1 \\
2 \\
1 \\
2 \\
1 \\
2\end{array}$} & \multirow{3}{*}{$\begin{array}{l}+69 \\
+52 \\
+43 \\
+27 \\
+79 \\
+65 \\
+53 \pm 6(\mathrm{~m} . \mathrm{e})\end{array}$} & $\begin{array}{r}79 \\
139\end{array}$ & $\begin{array}{l}2 \\
1\end{array}$ & $\begin{array}{l}+62 \\
+38\end{array}$ \\
\hline & & & \multicolumn{3}{|c|}{ Visual Binaries } \\
\hline & & & $\begin{array}{r}170 \\
64 \\
113 \\
145\end{array}$ & $\begin{array}{l}1 \\
1 \\
1 \\
2\end{array}$ & $\begin{array}{l}-35^{*} \\
+131 \\
+52 \\
-\quad 3 \dagger\end{array}$ \\
\hline
\end{tabular}

* Probably not a physical pair

† DB star; possible Stark-effect shift of $\mathrm{He}_{\mathrm{I}}$

Now assume that $|W| \leq 50 \mathrm{~km} / \mathrm{sec}$, i.e., about twice the dispersion in $W$. We find a range for values of the gravitational redshift, $\Delta k_{i}$, from

$$
\Delta k_{i}=\rho_{i}+\left(W_{0} \pm 50\right) \frac{d \rho}{d W} .
$$

Here $W_{0}$ and $d \rho / d W$ are from tables in Papers I and III, and we use the plus or minus sign that gives the smallest positive value of $\Delta k_{i}$. Clearly, this can only be done for stars not too far from the galactic polar caps; otherwise $d \rho / d W$ becomes too large. The ambiguity of sign of the maximum $W$ velocity results in a possible range of $100 d \rho / d W$, if we force the true $|W|$ to be an extremal, i.e., $50 \mathrm{~km} / \mathrm{sec}$. The footnote to Table 1 lists some stars providing reasonable estimates of the gravitational shift. They initially had $|W|>50$; from equation (2) a positive $\Delta k_{i}(\mathrm{~min})$ was found, such that $|W|=50$, with unchanged sign. The mean value, $\Delta k_{i}(\mathrm{~min})=+39 \pm 6 \mathrm{~km} / \mathrm{sec}$ (average deviation).

\section{MEAN REDSHIFTS}

Table 2 gives redshifts for individual stars. From Table 1, we obtained the mean redshift of Hyades DA stars directly. The extended Hyades group members give data that are less certain, but have expected velocities from Table 6 of Paper I and redshifts listed 
in our Table 2 ; the differences in velocity between white dwarfs and their late-type companion in visual binaries are also listed. Further late-type primaries in visual binaries are known, and a program of measuring velocity shifts and setting up standard wavelengths for the M-dwarf companions is under way; perhaps ten more redshifts can eventually be found from such wide binaries. With few exceptions, these common-proper-motion pairs are so wide that mass determinations will be only statistical in nature. The unweighted mean redshift of the sixteen spectra of twelve stars listed in Table 2 is $+51 \mathrm{~km} / \mathrm{sec}$.

A statistical estimate of the mean gravitational redshift for all the white dwarfs can be obtained from the entire material. From Paper III, the mean tangential speed of 110 white dwarfs we have studied, omitting the Hyades, is about $35 \mathrm{~km} / \mathrm{sec}$, so that the expected radial mean speed is about $25 \mathrm{~km} / \mathrm{sec}$. After correction for the radial component of solar motion (second line of column II of Table 1) we have fifty-three values of the quantity $\rho_{i}(0)+k_{i}$. (Omitting the Hyades from the sum, we would expect a mean error in $K^{\prime}$ to be of the order of $30 \times(47)^{-1 / 2}$, or $\pm 4.3 \mathrm{~km} / \mathrm{sec}$ if the measuring errors were zero.) The mean values of $K^{\prime}$ obtained are listed in Table 3 , together with the quantity "A.D.," the average deviation of each of the means. Note that the average deviations of the means are larger than expected; measuring error should contribute

TABLE 3

Statistical VALUe of RedShifT (KM/SEC)

\begin{tabular}{|c|c|c|c|c|c|}
\hline Group & $K^{\prime}$ & A D. & $n$ & $\Delta K^{\prime}$ & $K$ \\
\hline $\begin{array}{l}\text { All stars } \\
\text { All, omitting Hyades } \\
\text { All DA } \\
\text { Hyades DA } \\
\text { DA, omitting Hyades } \\
\text { DB. } \dot{D A} \dot{\text { F }} \dot{\mathrm{DG}} . \\
\text { DO; . }\end{array}$ & $\begin{array}{l}+501 \\
+461 \\
+602 \\
+56 \\
+562 \\
+30 \\
+27\end{array}$ & 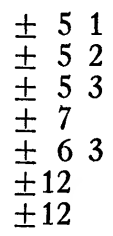 & $\begin{array}{r}52 \\
46 \\
37 \\
6 \\
31 \\
6 \\
9\end{array}$ & $\begin{array}{r}+37 \\
+41 \\
+54 \\
+63\end{array}$ & $\begin{array}{l}+538 \\
+504 \\
+656 \\
+56 \\
+625 \\
+30 \\
+27\end{array}$ \\
\hline
\end{tabular}

about $\pm 3 \mathrm{~km} / \mathrm{sec}$ to the spread expected from the intrinsic dispersion in radial velocity. In particular, the yellow DAss star with large proper motion, LP9-231, EG 199, has the extraordinarily high radial velocity of $-154 \mathrm{~km} / \mathrm{sec}$; corrected for normal solar motion, it has a velocity of $-145 \mathrm{~km} / \mathrm{sec}$. Consequently, it deviates by about $-200 \mathrm{~km} / \mathrm{sec}$ from the mean. Therefore, we computed the effect, $\Delta K^{\prime}$, of omitting the star from the various means formed and recomputed $K=K^{\prime}+\Delta K^{\prime}$. It is these $K$ values, without primes, in the last column of Table 3 that will be used henceforward; the column $n$ gives the number of stars in each tabulated $K$.

We have chosen a final value of $K=+51 \mathrm{~km} / \mathrm{sec}$ in correcting observed velocities in Table 1, as already mentioned. But in astrophysical discussion we must omit the single high-velocity star, EG 199 (LP9-231). The Hyades $K$ value of $+56 \mathrm{~km} / \mathrm{sec}$, or the gross $K$ mean of DA stars, $+66 \mathrm{~km} / \mathrm{sec}$, suggests that a mean gravitational red shift, $K$, lies in the range +55 to $+65 \mathrm{~km} / \mathrm{sec}$. Velocities of poor quality were given for record only in Table 4. The mean uncorrected value of $\Sigma_{\rho_{i}}$, for all thirty-nine stars listed in Table 4 , is $+42 \mathrm{~km} / \mathrm{sec}$. Since it included ten stars with peculiar spectra (like those with smaller shifts in Table 3), the mean from poor plates agrees with the primary data of Tables 1 and 3.

There are other genuine systematic effects-for example, the relatively large intrinsic, positive radial velocity of the Hyades stars (whose unweighted properties are given in Table 3), the non-uniform distribution of objects around the sky, and the apparently small redshifts of stars of type DB and late spectral type. Negative line shifts caused by pressure broadening, or quadratic Stark broadening, and the effects of for- 
bidden components on measured He I wavelengths may explain systematic effects in Table 3.

\section{CRITIQUE}

In general, the mean redshift derived is well enough determined to establish the reality of the Einstein gravitational shift and the magnitude of the gravitational potential in the layers forming the hydrogen lines. A few methodological questions should be first disposed of. As far as we know, there is no reason to expect asymmetries and velocity shifts (Griem 1964) in Balmer lines broadened by Stark effect, until quasi-molecules or multibody collisions occur. Factors like quadratic Stark effect and the asymmetrical $\left(\lambda-\lambda_{0}\right)^{4}$ term, are large only in the extreme wings; ion broadening acts differently on

TABLE 4

ObServed Velocities with POOR INTERnal Agreement: For ReCORd ONLy

\begin{tabular}{|c|c|c|c|c|c|c|c|c|}
\hline \multicolumn{3}{|c|}{$\mathrm{KM} / \mathrm{SEC}$} & \multicolumn{3}{|c|}{$\mathrm{KM} / \mathrm{SEC}$} & \multicolumn{3}{|c|}{$\mathrm{KM} / \mathrm{SEC}$} \\
\hline $\mathrm{EG}$ & $\rho$ & Remarks* & EG & $\rho$ & Remarks* & EG & $\rho$ & Remarks* \\
\hline $\begin{array}{r}166 \\
8 \\
13 \\
18 \\
20 \\
22 \\
29 \\
34 \\
38 \\
47 \\
63 \\
65 \\
77 \\
84\end{array}$ & $\begin{array}{l}+80 \\
+\quad 7 \\
+16 \\
+114 \\
-53,+111 \\
+134 \\
+103 \\
-105 \\
+159 \\
+116 \\
+30 \\
+96\end{array}$ & $\begin{array}{l}\text { L } \\
\text { F } \\
\text { L } \\
\text { F } \\
\text { E } \\
\text { DD } \\
\text { L } \\
\text { F } \\
\text { E, DD } \\
\text { L } \\
\text { D } \\
\text { L } \\
\text { P } \\
\text { L }\end{array}$ & $\begin{array}{r}86 \\
90 \\
93 \\
94 \\
97 \\
111 \\
192 \\
114 \\
174 \\
115 \\
117 \\
118 \\
120 \\
121\end{array}$ & $\begin{array}{l}+15,+94 \\
+152 \\
+58 \\
+49 \\
+8 \\
+23 \\
+83 \\
+51 \dagger \\
+57 \\
+43 \\
+131 \\
+10 \\
+55 \\
+22\end{array}$ & $\begin{array}{l}\text { DD } \\
\text { wk } \\
\text { P } \\
P \\
P \\
D \\
F \\
P \\
F \\
D \\
D \\
D, w k \\
D \\
P\end{array}$ & $\begin{array}{l}123 \\
175 \\
176 \\
200 \\
127 \\
132 \\
135 \\
137 \\
150 \\
155 \\
158 \\
159 \\
161\end{array}$ & $\begin{array}{l}-\quad 9 \\
-50 \\
-\quad 77 \\
+53 \\
+46 \\
+48 \\
+\quad 81 \\
+35 \\
+16,+108 \\
+94 \\
+5,+45 \\
+34 \\
-154\end{array}$ & $\begin{array}{l}\text { wk } \\
\text { F } \\
\text { L, wk } \\
\text { F } \\
\text { wk } \\
\text { P } \\
\text { F } \\
\text { F } \\
\text { DD } \\
\text { F } \\
\text { F, DD } \\
\text { F } \\
\text { L, wk }\end{array}$ \\
\hline
\end{tabular}

* $\mathrm{P}=$ poor plate; $\mathrm{L}=$ low dispersion $(380 \AA / \mathrm{mm}) ; \mathrm{F}=$ fair plate; $\mathrm{E}=$ emission lines, interfere with absorption; wk, weak lines; $\mathrm{D}=$ discordance between direct and reverse large; $\mathrm{DD}=$ discordance between different plates large For $\mathrm{EG} 20$, $87, V_{\text {abs }}>V_{\text {em }}$

$\dagger$ The primary of EG 114 has $\rho=-4 \mathrm{~km} / \mathrm{sec}$

alternate members of the Balmer series, producing a small redshift of the zero Starkshift central component. Popper (1954) expected higher-order terms in the shifts to be less than $1 \mathrm{~km} / \mathrm{sec}$. The cores, formed at lower pressures, have even smaller shifts. Weidemann, in a private communication, suggests, however, that $\mathrm{H} \gamma$ might have a redshift of as much as $15 \mathrm{~km} / \mathrm{sec}$. This possibility needs detailed computation of the core and wing, and a study of what is really measured, what weight is given to the core and to the inner parts of the wings. For symmetric lines such questions are irrelevant.

The existence of helium lines in DB stars reflects such a large $\mathrm{He} / \mathrm{H}$ ratio, that $\mathrm{He}+$ $\mathrm{He}$ collisions could dominate, rather than $\mathrm{He}+\mathrm{H}^{+}$, or $\mathrm{He}+e^{-}$. In addition, $\mathrm{He}$ Ir line of the Pickering series, if present and blended with $\mathrm{H}$, would cause a negative shift in DO stars. In the DB stars each of the lines has a different profile and peculiar shifts and erroneous velocities may well be expected. To study the $\mathrm{He}$ I shifts we have observational material which included seventeen plates of ten DB stars and forty complete measures of these plates. The mean velocity for each measurement of a plate was obtained, and the residuals tabulated for each line and averaged. Table 5 shows the systematic displacements between different helium lines. No information on the mean shift of all the lines can be obtained by this method. Table 3 shows that the mean redshift of the 
DB stars was $+30 \mathrm{~km} / \mathrm{sec}$ and of the DA stars near $+60 \mathrm{~km} / \mathrm{sec}$, i.e., a systematic mean displacement of about $-0.4 \AA$ for $\mathrm{He}$. Table 5 gives the wavelength; the series notation; $\Delta v$, the mean residual in $\mathrm{km} / \mathrm{sec}$; and, $n$, the number of measurements.

There is little doubt that the strong diffuse triplets of $\mathrm{He} I, 2^{3} P^{o}-n^{3} D$, show a negative shift as compared to most other lines. It is possible that the ${ }^{1} S-n^{1} P^{o}$ has a negative shift also. This series has sharp lines and is subject to a relative overpopulation in B stars with metastability effects; the $2^{3} P^{o}-n^{3} D$ also shows such overpopulation effects but less so than does $\lambda 3888$ from the $2^{3} S$ level. The more likely explanation is the complex of pressure broadening and shifts, and the static Stark effect for the diffuse triplets. For example, the forbidden transitions at $\lambda \lambda 4024,4470$ (the $2^{3} P^{o}-n^{3} F^{o}$ lines) appear in main-sequence B stars. Presumably they contribute even more and cause violet shifts, since they are unresolved from $\lambda \lambda 4026,4471$ in the DB stars. With lines pressure broadened to half-widths of $20 \AA$, line shifts in the wings of the order of $4 \AA$ are quite possible.

TABLE 5

SHIFTS OF INDIVIDUAL LINES OF HE I IN DB STARS

\begin{tabular}{|c|c|c|c|c|c|}
\hline & $\lambda$ & Series & $\Delta \mathrm{v}(\mathrm{km} / \mathrm{sec})$ & $n$ & Remarks \\
\hline $\begin{array}{l}4922 \\
4388 \\
4144\end{array}$ & - & $\begin{array}{l}2^{1} P^{o}-4^{1} D \\
2^{1} P^{o}-5^{1} D \\
2^{1} P^{o}-6^{1} D\end{array}$ & $\begin{array}{l}+130 \\
+17 \\
+19\end{array}$ & $\begin{array}{r}6 \\
16 \\
2\end{array}$ & $\begin{array}{l}\text { On sloping continuum } \\
\text { Some blend } \lambda 4121\end{array}$ \\
\hline $\begin{array}{l}5016 \\
3965 \\
3613\end{array}$ & & $\begin{array}{l}2^{1} S-3^{1} P^{o} \\
2^{1} S-4^{1} P^{o} \\
2^{1} S-5^{1} P^{o}\end{array}$ & $\begin{array}{l}+66 \\
-28 \\
-254\end{array}$ & $\begin{array}{r}1 \\
23 \\
1\end{array}$ & $\begin{array}{l}\text { On sloping continuum } \\
\text { Low accuracy, weak }\end{array}$ \\
\hline $\begin{array}{l}4713 \\
4121\end{array}$ & & $\begin{array}{l}2^{3} P^{o}-4^{3} S \\
2^{3} P^{o}-5^{3} S\end{array}$ & $\begin{array}{r}+26 \\
+146\end{array}$ & $\begin{array}{r}21 \\
9\end{array}$ & Bad blend $\lambda 4144$ \\
\hline $\begin{array}{l}4471 \\
4026 \\
3819\end{array}$ & . & $\begin{array}{l}2^{3} P^{0}-4^{3} D \\
2^{3} P^{o}-5^{3} D \\
2^{3} P^{o}-6^{3} D\end{array}$ & $\begin{array}{l}-31 \\
-18 \\
-40\end{array}$ & $\begin{array}{l}34 \\
33 \\
18\end{array}$ & \\
\hline 3888 & & $2^{3} S-3^{3} P^{o}$ & +10 & 32 & \\
\hline
\end{tabular}

Again the cores should be less shifted. Griem (1964) gives some theoretical values of the He I shift, $d$, in units of the half-half-widths, $w$. Not all series are tabulated in his work; however, $d / w$ is negative, averaging -0.31 for $\lambda \lambda 3965,3613$. It is negative for $\lambda 5876$, and equal to -0.23 ; this is a member of the $2^{3} P^{0}-n^{3} D$ series, like $\lambda \lambda 4472,4026$ (which he omits). On the other hand, it is positive for $\lambda \lambda 4922,4388(\approx+0.13)$ and $\lambda 4713$ $(\approx+0.95)$. The signs correlate with the roughly determined residuals in Table 5 . Although the details are not worth pursuing, the existence of systematic effects in the velocities and therefore in the deduced gravitational shifts of the DB stars is very probable. Collisions of $\mathrm{He}+X$ often displace lines of element $X$ to the violet (Chen and Takeo 1957). If we consider metallic lines, $d / w$ is positive for $\lambda 3933$ of $\mathrm{Ca}$ II, but the broadening parameter is very small; $\lambda 3835$ of $\mathrm{Mg}$ I has negative $d / w$ and considerable broadening, so that it could have a negative shift. As an example, we have EG 119, Ross 640 , which has a strong $\mathrm{Mg}$ I line, the Ca II lines, and a residual, $\rho_{i}-K^{\prime}=-28 \mathrm{~km} /$ sec. In case the atmospheres of metallic-line white dwarfs contain helium rather than hydrogen, the broadening and shift theory will be grossly different than for a hydrogenrich star. Only the DA stars can, at present, be used for quantitative evaluation of the gravitational shift.

The statistical technique to evaluate $K$ has been very elementary, and would be correct only if the stars were uniformly distributed over the sky and no velocity groups 
existed. Non-uniformity arises from two selection effects: the stars in the Hyades were observed preferentially, and many white dwarfs have been found in the north galactic pole region. The observed radial velocity is resolved into components: $\rho_{i}(0)$, the true radial velocity with respect to the Sun, $k_{i}$ the gravitational redshift, and $V \odot \cos \lambda$, the projected solar motion with respect to the local standard of rest of low velocity stars. The observed velocity $\rho_{i}$ is

$$
\rho_{i}=\rho_{i}(0)+k_{i}+V \odot \cos \lambda .
$$

If we average over a uniform distribution,

$$
\left\langle\rho_{i}\right\rangle=\left\langle\rho_{i}(0)\right\rangle+\left\langle k_{i}\right\rangle-V \odot\langle\cos \lambda\rangle,
$$

which should reduce to

$$
\left\langle\rho_{i}\right\rangle=\left\langle k_{i}\right\rangle=K
$$

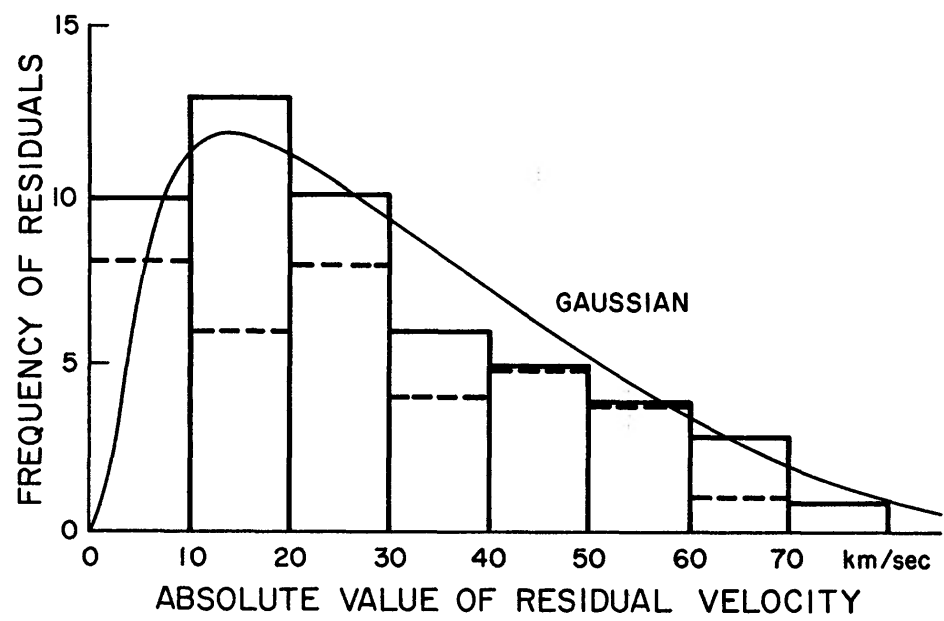

Fig. 1.-The histogram (solid lines) gives the frequency of residuals, $|\delta v|$, from Table 1 (excluding EG 199) corrected for solar motion. The mean redshift was $+51 \mathrm{~km} / \mathrm{sec}$. The curve is a Gaussian with an average deviation equal to that of the total sample of 52 stars. The dashed line is the histogram for DA stars only, giving residuals, $\left|\delta v_{A}\right|$, with respect to their mean $K$ of $62 \mathrm{~km} / \mathrm{sec}$.

The non-uniformity of the distribution over the sky can be estimated by summing $\left\langle\rho_{i}\right\rangle$ without correction for solar motion for all the thirty-one DA stars. The result is $+\mathbf{5 5 . 9}$ $\mathrm{km} / \mathrm{sec}$, excluding the Hyades, and $+62.0 \mathrm{~km} / \mathrm{sec}$ for thirty-seven stars including them. These values of $K$ differ by $-6 .($ and $-3.6 \mathrm{~km} / \mathrm{sec}$ from those given in Table 3 . The mean value of the solar-motion correction was only $+4 \mathrm{~km} / \mathrm{sec}$ for the DA stars. No reasonable change of the apex, or of $V \odot^{\prime}$ can produce a large change in $K$. An undetected group of stars with large systematic motion could, however, produce errors in $K$. For example, in Paper I we discussed the Wolf 219 group, whose seven possible white dwarf members would have a large negative radial velocity if their motions are made parallel. Fortunately for our present result, no members of the Wolf 219 group have measurable radial velocities, since they are almost all of type DC or $\lambda 4670$ !

\section{INTERPRETATION OF THE OBSERVATIONS}

The last line of column II in Table 1 gives the deviation of each radial velocity from the mean, $\rho_{i}-K^{\prime}$. This quantity may be affected by line shifts but otherwise gives an estimate of the radial-velocity dispersion of the white dwarfs. Figure 1 is a histogram displaying the absolute values of the residuals corrected for solar motion $|\delta v|$; they are 
$\left|\rho_{i}-K^{\prime}-V \odot \cos \lambda\right|=|\delta v|$ for most stars, but $\left|\rho_{i}-K^{\prime}-V_{0}\right|=|\delta v|$ for binaries and cluster members, where $V_{0}$ is the binary companion or cluster velocity. Star LP9231 , as usual, is omitted. The average deviation is $\langle|\delta v|\rangle=27.3 \mathrm{~km} / \mathrm{sec}$. The measuring errors were estimated to be smaller $( \pm 13 \mathrm{~km} / \mathrm{sec}$ for a good velocity). The radial velocities show that, on the whole, the white dwarfs are a moderate velocity population, in agreement with the $U, V$ components shown in Papers I and III.

These residuals are somewhat less meaningful than those derivable from a restricted sample of the DA stars alone. There is a systematic difference in $K$ of $11.2 \mathrm{~km} / \mathrm{sec}$, in Table 3, between all stars and all DA stars. We therefore computed $\left|\delta v_{A}\right|=\mid \rho_{i}-$ $V \odot \cos \lambda-62 \mid$. Even though the material is reduced in size, the histogram of $\left|\delta v_{A}\right|$ in Figure 1 (shown in dashed lines) gives about the same distribution, with an average deviation $\left\langle\left|\delta v_{A}\right|\right\rangle=28.1 \mathrm{~km} / \mathrm{sec}$, near our first value, even though this is a more physically coherent group. A few high-velocity objects are easily detected, in addition to LP9-231. For example, EG 4 has a $U$ component of either +114 or $+74 \mathrm{~km} / \mathrm{sec}$, and a $\delta v$ of +57 ; EG 58 has an unusually large $W=-82 \mathrm{~km} / \mathrm{sec}$ and $\delta v$ of $-63 \mathrm{~km} / \mathrm{sec}$; EG 151 has $(U, W)$ of either $(-51,-63)$ or $(-35,-49) \mathrm{km} / \mathrm{sec}$, and $\delta v=+62 \mathrm{~km} /$ sec. The DG star, vMa 2, EG 5 , has a much smaller velocity than originally published. The residual $\delta v=+3$, is very small compared to $(V, W)$ of $(-83,-70) \mathrm{km} / \mathrm{sec}$. But this star may well have its metallic lines (Ca II, Fe I) moved to shorter wavelengths as expected for helium-metals collisions. To make $\delta v \approx 50 \mathrm{~km} / \mathrm{sec}$, the pressure shift would have to be about $1 \AA$ to the violet. Weidemann (1960) has discussed the problem of the opacity and pressure in vMa 2, and the expected velocity shifts of the metallic lines.

The gravitational redshift for a star is obtained from

$$
K=0.635 \mathrm{~km} / \mathrm{sec}(M / M \odot)(R / R \odot)^{-1} .
$$

The observed range within which $K$ should lie is +55 to $+65 \mathrm{~km} / \mathrm{sec}$. The mean $K$ gives the mean value of $(M / R)$ in solar units. Since the degenerate-gas law provides a locus of $M / R$, for a given composition, the observations give $M$ and $R$, for an assumed composition, or the composition and $M$, if $R$ is determined by photometry, as was done in Papers I and III. The mean radius of the lower sequence was $0.01 R \odot$, so that $M / M \odot \approx 0.9-1.1$. Since stars of so large a mass would be near the Chandrasekhar limit, if their mean atomic weight was that of $\mathrm{He}^{4}$ (i.e., $\mu_{e}=2$ ) the statistical treatment will have to be done more carefully.

We have therefore attempted to re-evaluate the radii from the $U B V$ colors, basically using the temperature calibration shown in Figure 6 of Paper I, and in more detail by Weidemann (1966), who gives the Balmer-line blanketing vectors for white dwarfs. These temperatures are very rough, especially at the high-temperature end; bolometric corrections for the white dwarfs are not accurately known. The radius is obtained from

$$
-\log R / R \odot=0.2\left(M_{v}+\Delta M_{b}\right)-2 \log \theta_{e}-1.06
$$

and is given in Table 1. The bolometric corrections were obtained from main-sequence models of Strom or Mihalas. The latter notes that $\Delta M_{b} \approx($ constant $-4.03 \log T$ ) at high $T$, so that the term $0.2 \Delta M_{b}$ becomes $+0.81 \theta_{e}$ and partially cancels the steep $-2 \log \theta$ variation in equation (7). These photometric radii are, of course, rendered ambiguous by the double-valued $\left(M_{v}, U-V\right)$ relation, (which may arise from differences in line blanketing and Balmer jump between stars). But we cannot, at present, correct for this, and consequently give the two possible values of $-\log R / R \odot$, in the last column of Table 1, together with $\theta_{e}$, the reciprocal effective temperature used to derive them.

The radial velocities and radii for the DA stars are plotted in Figure 2; the open circles represent stars for which the $M_{v}$ is not uniquely determined from parallax, companions, or cluster membership. The scatter is very large; negative velocities are omitted. In Figure 2, $A$ (upper sequence, bright $M_{v}$ ) these uncertain cases appear at the left, in 
Figure 2, $B$ at the right. Since many have large radial velocities, Figure 2, $A$ gives the unexpected appearance of a redshift that decreases as the photometric radius becomes smaller, unexpected and probably untrue. In Figure 2, $B$, there is no clear trend; the internal consistency is improved if most stars fall on the lower sequence. In Figure $2, B$, we find that the median value of $k_{i}$ is $+58 \mathrm{~km} / \mathrm{sec}$, and of $-\log R / R \odot=1.97$, i.e., $0.0107 R \odot$. This median velocity is below the mean of all DA stars $+66 \mathrm{~km} / \mathrm{sec}$ (Table 3 ), not at all unexpected in a skew distribution of redshift velocities which permits no negative values to be included. A few DA, F are included, and obviously LP9-231 is omitted because of its large negative velocity. The mass derived from the median values of $k_{i}$ and $R$ is $0.98 M \odot$, almost certainly too high. Whether we should use the mean, harmonic mean, or median radius is not worth discussing in detail here, especially with the uncertainty in luminosity for seventeen of the thirty-seven stars.

Deriving temperatures and radii from colors and luminosities has many uncertainties. A more detailed study of line blanketing by Weidemann (1963) gives somewhat different median radii. He used older luminosities, the line-blanketing vectors based on actual equivalent widths for twenty-two DA stars and $U B V$ colors for five more. The median value of $-\log R / R \odot$ he derived is 1.91 ; a slightly larger radius corresponding to a change of $0.3 \mathrm{mag}$ in $M_{v}+\Delta M_{b}$ (eq. [7]), or of 0.03 in $\theta_{e}$.

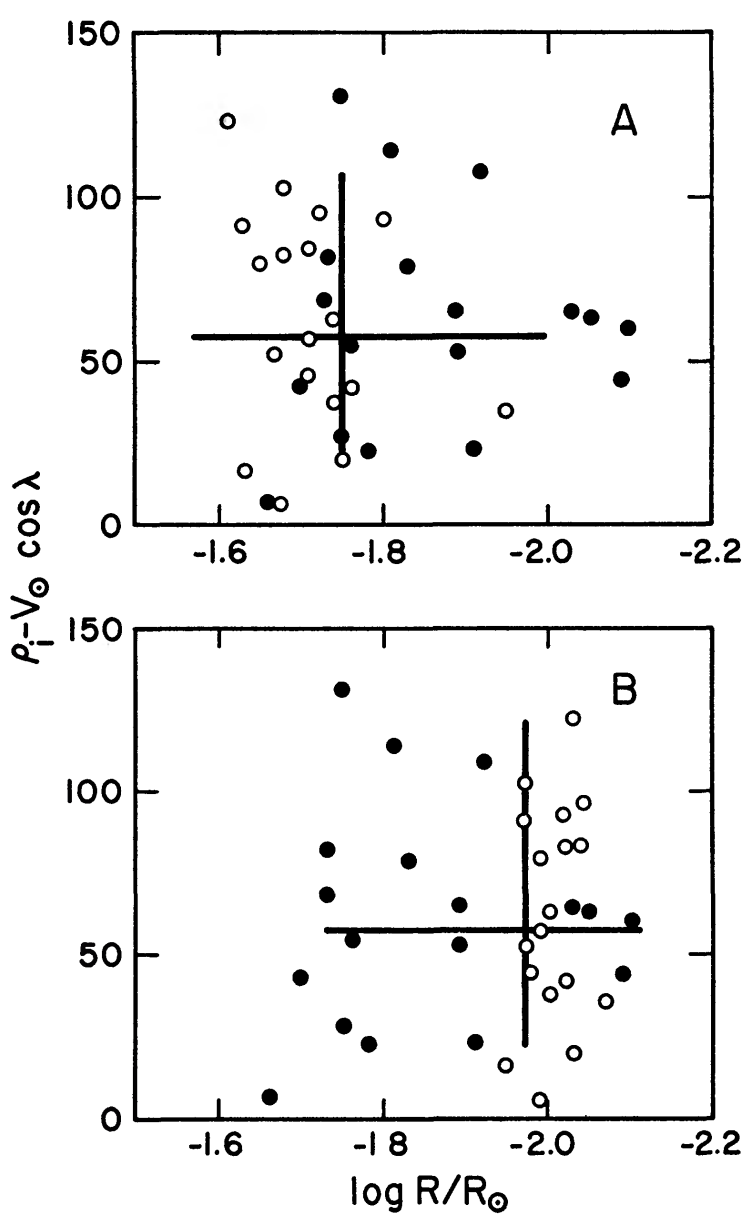

FIG. 2.-The individual velocities of hydrogen-line, DA-type white dwarfs, corrected for normal solar motion, and the photometrically derived radii as solid dots. $(A)$ The upper diagram shows the stars of uncertain radii (open circles) plotted with their luminosities from the upper sequence. $(B)$ The lower diagram shows the radii from the lower sequence (open circles). The median photometric radius, for this assumption, and median redshift are shown. 
The theoretical dependence of redshift on mass for zero-temperature degenerate configurations can be obtained from Hamada and Salpeter (1961). Their results differ from the Chandrasekhar models only slightly and are available for various compositions. For $\mathrm{He}^{4}\left(\mu_{e}=2\right)$ and a moderate mass their radii are about 3 per cent smaller than Chandrasekhar's. At $0.9 M \odot$ the predicted radius changes from $0.0085 R \odot$ for $\mathrm{He}^{4}$ to $0.0061 R \odot$ for $\mathrm{Fe}^{56}$ to $0.0055 R \odot$ for the composition at nuclear equilibrium. Redshifts at a given mass can differ by a factor of 40 per cent, depending on composition. The median photometric radius and redshift from Figure $2, B$,is replotted in Figure 3, with a reasonable spread corresponding to plausible ranges of error. We have also plotted in Figure 3

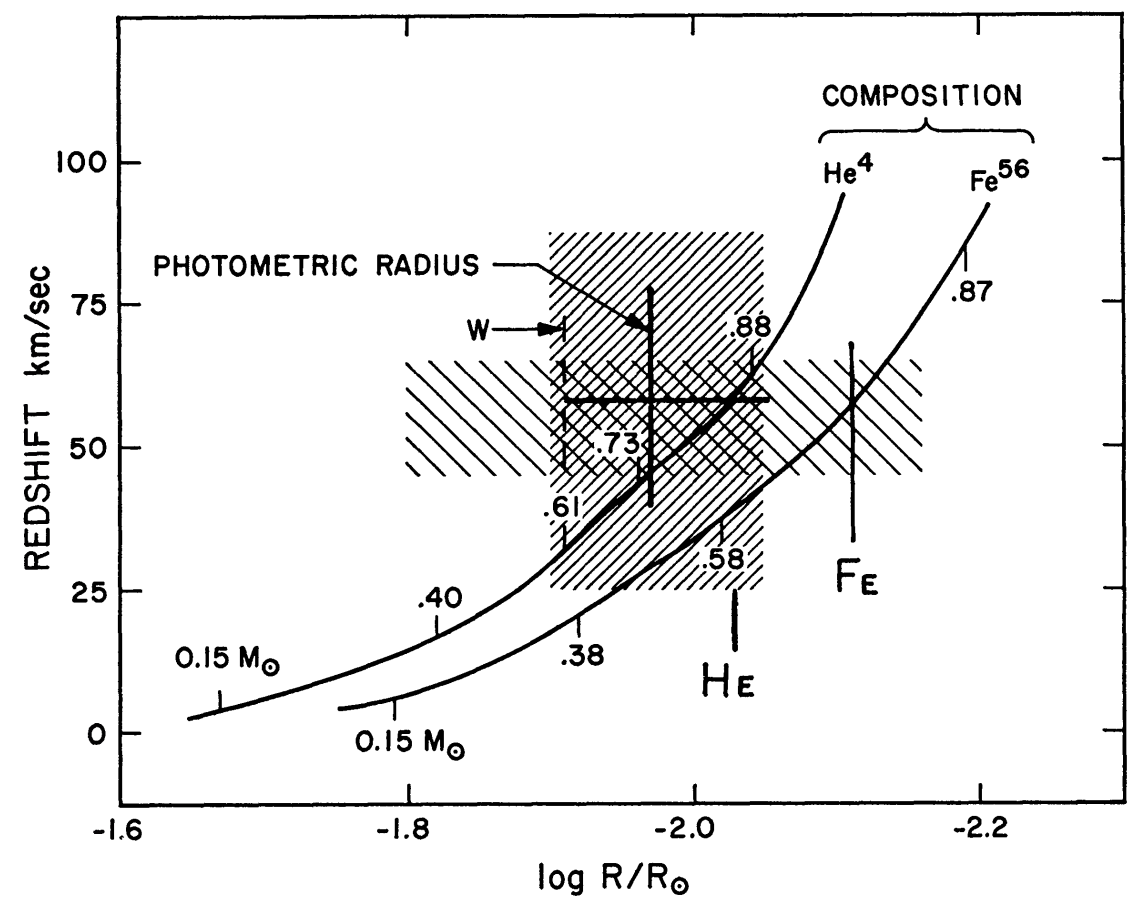

FIG. 3.-The theoretical redshift depends on mass and composition; the theoretical curves, labeled with masses, are for $\mathrm{He}^{4}$ and $\mathrm{Fe}^{56}$ (from Hamada and Salpeter). The observed median redshift and radius of DA stars is shown as a cross with a hatched zone showing plausible spreads in both coordinates. The median radius, as given by Weidemann, is the vertical dashed line labeled " $W$." The two vertical lines marked $\mathrm{He}$ and $\mathrm{Fe}$ are the radii given by the redshift and the theoretical mass-radius relation. They correspond to a median mass of $0.86 M \odot$ for a helium core and $0.73 M \odot$ for an iron core.

curves giving the expected redshifts for cold degenerate stars with $\mathrm{He}^{4}$ and $\mathrm{Fe}^{56}$ cores and no envelopes. At a given mass, the $\mathrm{Fe}^{56}$ star has the smaller radius and therefore the larger shift. The photometrically derived median log radius and the observed redshift lie near the theoretical position of a $\mathrm{He}^{4}$ star of mass $0.78 \mathrm{M} \odot$. A steep increase of redshift with decreasing radius is expected, for either composition, and is not seen in the scatter of observed points plotted in Figure 2, $A$ and 2, $B$. This certainly does not mean that the mass-radius relation is not confirmed-merely that the observational data is not sufficiently good. The theoretical slope, near the median log $R$, is $230 \mathrm{~km} / \mathrm{sec}$ per unit change in $\log R$. The observational uncertainty, in luminosity alone, produces an uncertainty in $\log R$ of \pm 0.16 , or in the redshift of $\pm 35 \mathrm{~km} / \mathrm{sec}$, per star.

It is interesting to note that both the $\mathrm{He}^{4}$ and $\mathrm{Fe}^{56}$ curves lie below the observed median; it is implausible that elements with $\mu_{e}<2$ (e.g., $\mathrm{He}^{3}, \mathrm{~T}^{3}$, or $\mathrm{H}^{1}$ ) exist in the interior. It seems most probable that the photometric radii derived from $\left(M_{v}, \theta\right)$ are too large. The median value of $\theta_{e}$ from DA stars in Table 1 is 0.47 and is most uncertain. 
We estimate the change in our temperature scale required to put the intersecting median lines of Figure 3 on the $\mathrm{He}^{4}$ curve as $\langle\Delta \theta\rangle=-0.03$, and on the $\mathrm{Fe}^{56}$ curve as $\langle\Delta \theta\rangle=$ -0.07 , which is an error of 6-15 per cent in temperatures. (In order to obtain this, we differentiate an approximation to the bolometric correction, to find that $\Delta \log R / R \odot \approx$ $0.85 \Delta \log \theta$ ). A possible systematic error in luminosity, $M_{v}$, would only have to be +0.25 mag to fit the $\mathrm{He}^{4}$ curve, and +0.65 mag to fit the $\mathrm{Fe}^{56}$ curve. Finally, if a systematic measuring error exists in the redshifts we give, it requires $-13 \mathrm{~km} / \mathrm{sec}$ to make the photometric radius fit the $\mathrm{He}^{4}$ curve and $-30 \mathrm{~km} / \mathrm{sec}$ to fit the $\mathrm{Fe}^{56}$ curve. The latter is almost certainly unacceptably large; we believe that systematic errors are more likely to be near $10 \mathrm{~km} / \mathrm{sec}$. A higher-order Stark effect that causes a positive pressure shift of more than $30 \mathrm{~km} / \mathrm{sec}$ would be needed to bring the observed median to the $\mathrm{Fe}^{56}$ curve. The temperature correction of $\Delta \theta=-0.07$ required to fit the $\mathrm{Fe}^{56}$ curve also seems to be unacceptably large. The effect on colors of lines other than hydrogen has not been included in our analysis and may be significant for the cooler stars (Weidemann 1966).

In résumé, we can obtain an approximate fit between median observed redshift and the theoretical mass-radius relation for a helium composition if the median mass of the white dwarfs is $0.78 M \odot$; if $\mathrm{Fe}^{56}$ is used the mass is $0.71 M \odot$. If we force an exact agreement between the theoretical mass-radius relation for a $\mathrm{He}^{4}$ interior and the observed redshift, we must raise the median temperature of the DA's from $10700^{\circ} \mathrm{K}$ to $12220^{\circ} \mathrm{K}$, thereby reducing the median radius to $\log R / R \odot=-2.03(0.0093 R \odot)$. The mass derived is then $0.86 M \odot$. If the hydrogen envelope is at all important, the resulting increase in radius would reduce the redshift expected at a given mass, and the above masses would be minimum values. The mean density (for $\mathrm{He}^{4}$, mass $0.87 \mathrm{M} \odot$ ), is $1.6 \times 10^{6} \mathrm{gm} /$ $\mathrm{cm}^{3}$, the central density $1.2 \times 10^{7} \mathrm{gm} / \mathrm{cm}^{3}$. The models fall well below the maximum possible mass of a white dwarf, with central densities low enough so that pycnonuclear or inverse-beta-decay reactions are slow. Individual stars of larger mass and central density may exist, nearer a limit for instability or collapse, but no such very large redshifts have yet been found.

If the core composition is assumed to be $\mathrm{Fe}^{56}$, the derived median mass is $0.73 \mathrm{M} \odot$. The central density is $1.9 \times 10^{7} \mathrm{gm} / \mathrm{cm}^{3}$, again far below the collapse point. In Figure 3 the discrepancy between the radius for the Fe-core models and the usually deduced value seems very large, and would require drastic revision of the temperature scale.

\section{A SPECULATION CONCERNING THE PARENT STARS}

The mass derived is appreciably higher than $0.56 M \odot$ found earlier by Greenstein (1958) from photometric radii alone, without knowledge of redshifts. Rough agreement between the observed redshift and the radius can be reached with the now reduced value of median radius and the $M, R$ relation slightly different from that given by Chandrasekhar. It has some interesting, if speculative, implications. Stars in the mass range below $1.4 M \odot$ (helium) or $1.01 M \odot$ (equilibrium, heavy-element composition) should eventually become degenerate. We find from the present masses of white dwarfs that on the average about 35 per cent of the mass has been lost from such stars in various evolutionary stages. The mass loss would have to be much larger for Population I white dwarfs. Our data weakly suggest that the residual material is more like $\mathrm{He}^{4}$ than $\mathrm{Fe}^{56}$, but it could, of course, be as heavy as $\mathrm{C}^{12}$ to $\mathrm{Mg}^{24}$ without contradicting our observations. If we believe that an appreciable fractional mass loss has occurred, it appears that the parent stars of the DA group almost certainly had masses greater than $0.78 M \odot$ (from approximate fit of redshifts) and probably greater than $0.86 M \odot$ (from redshifts, altered photometric radii, and theoretical mass-radius relation). If any appreciable fraction of the DA sample belongs to the very old disk or halo population, stars of these populations now evolving should also have masses $\approx 0.9 M \odot$. Interpretation of the globular cluster, horizontal-branch (HB) stars by Faulkner (1966) has suggested two possible sets of evolutionary tracks for such stars. In one, the original helium content in 
the interior of the HB stars was low, 10 per cent, and the mass is $1.25 M \odot$. In another the helium content was high, 35 per cent, and the mass of the HB stars is $0.75 M \odot$. Similar arguments apply to stars at the turnoff point in globular clusters. The present observations suggest a larger median mass than previously, and one above $0.75 M \odot$.

Does any appreciable fraction of the DA stars belong to the halo of very old disk populations? A count in Table 1 of the $U, V$ velocities shows that nine DA stars have either $\left(U^{2}+V^{2}\right)^{1 / 2} \geq 50 \mathrm{~km} / \mathrm{sec}$ or $W \geq 30 \mathrm{~km} / \mathrm{sec}$, while thirteen have small space motions. (In the latter we count the Hyades as one star and adopt the smaller of the two space motions, where ambiguity exists.) Consequently, nearly half of our sample is very old Population I or Population II. Had the mean redshift indicated a mass near $0.4 M \odot$, as in 40 Eri $\mathrm{B}$, this type of argument would have been impossible. But the presently derived median mass of the DA stars is closer to that of Sirius B than of 40 Eri B! The high mass corresponding to the median redshift provides a weak indication favoring a low primeval helium content.

The masses and the parent stars may be atypical in that velocities can only be measured for white dwarfs with strong hydrogen or helium lines. In Paper I it can be seen that the DC or $\lambda 4670$ stars populate mainly the lower sequence (smaller radius, larger mass). The late-type, metallic-line stars are rare, it appears, and intrinsically faint, and are not represented in our measurements.

\section{APPENDIX}

\section{AN ASTROPHYSICAL PUZZLE}

The calibration of the scale of photometric radii used here is based essentially on the equivalent black-body temperatures for near-black-body white dwarfs, which gives about the same temperature scale as $\theta_{e}(W)$ (Weidemann 1963; denoted hereafter as "W"). An interesting new study using more elaborate model atmospheres and fitting both $U B V$ colors and $\mathrm{H} \gamma$ and $\mathrm{H} \delta$ line profiles was carried out by Terashita and Matsushima (1966). We were aware of this investigation (denoted hereafter as "TM") but cannot now reconcile our photometric radii, deduced masses, or the observed large redshift with the change in temperature scale given by TM. The essential result is that $\theta_{e}(\mathrm{TM})-\theta_{e}(W)=-0.06$, and $\theta_{e}(\mathrm{TM})-\theta_{e}(\mathrm{GT})=-0.10$. In addition, TM derive a mean $\log g=+7.0$ from $\mathrm{H} \gamma$ line profiles, while our median redshift and the theoretical $M, R$ relation gives $\log g=+8.4$. The mass seems far too low if derived from the TM scale of temperature and $\log g$. In determining the mass, the use of either mean or median values gives little difference in the result:

$$
\log \frac{M}{M_{\odot}}=-0.4 M_{b}+4 \log \theta_{e}+\log g-2.32 .
$$

The partial cancellation of sensitivity to $\theta_{e}$ is the same as in equation (7). The thirty-five stars for which our Table 1 gives radii and luminosities (choosing the lower sequence) have median $M_{b}=+11.4$, mean $=+10.9$, and median $\theta_{e}=0.475$ and mean $=0.428$, according to GT. The resulting "photometric" masses depend on $g$. If we use the spectroscopic mean log $g=+7.05$, according to TM and our temperature scale, the mean $M=0.074 M \odot$, obviously too small, if our temperatures are correct. There are only a few stars in common between TM and GT, so that we proceed by taking data from either source. Let us temporarily accept a systematic correction of -0.10 in $\theta_{e}$ in our Table 1 and compute $M_{b}$ with the new bolometric correction. Then the median $\theta_{e}=0.37$ and $M_{b}=+10.9$; with the TM value of surface gravity, the spectroscopically derived mass is $M=0.47 M \odot$. The redshift is expected to be only $20 \mathrm{~km} / \mathrm{sec}$, close to 40 Eri B and far less than observed.

The discrepancy is so large that it is not certain that the model-atmosphere approach has been completely successful. The TM models still neglect Balmer- and Lyman-line blanketing 
and have a much lower value of $T_{0} / T_{e}$ than is given by more approximate, gray-body computations by Weidemann or Uesugi (1962). It is also noteworthy that Figure 4 of TM shows that even at $\log g=9$, very high pressures, they predict $U-B$ colors about -0.15 mag more negative than the usual black-body calibration has ever done. In addition, none of the line-free or weak-line stars are observed so far above the usual black-body line. The TM estimates of $\theta_{e}$ and $\log g$ from colors alone, excluding DAwk (weak hydrogen line) stars, give a median log $g=+7.3$ and a median $\theta_{e}=0.35$. With our median luminosity, the change in bolometric correction and temperature results in a mass, from equation (A1), of $0.68 M \odot$, which corresponds to a redshift of $38 \mathrm{~km} / \mathrm{sec}$. This value is within the range of possibility. Note that the work of TM (their Fig. 5) results in a clear dependence of $g$ on $\theta_{e}$ for most DA stars, which seems implausible as a physical phenomenon in a group of stars cooling at constant radius. The astrophysical problem is to locate the best value of $\theta_{e}$, when $\theta_{e}$ and $g$ combine to affect the observable long-baseline color, Balmer discontinuity, and $\mathrm{H} \gamma$ profile. Finally, note that TM assume the $\mathrm{H} / \mathrm{He}$ abundance ratio of 7 ; it is possible that DA stars are richer in helium, which would reduce the opacity. Consequently, if we add helium, the situation is worse, since the same models would have resulted but with still lower values of the surface gravity.

Matsushima (1966) has reconsidered the effect of additional opacity sources, in the ultraviolet, on his model atmospheres. He believes that such unknown opacities may have caused the erroneous colors and low surface gravities of the TM white-dwarf models.

\section{REFERENCES}

Chen, S Y., and Takeo, M. 1957, Rev. Mod. Phys, 29, 20.

Delhaye, J. 1966, Galactic Structure, ed. A. Blaauw and M. Schmidt (Chicago: University of Chicago Press), $\mathrm{p} 71$.

Eggen, O. J., and Greenstein, J. L. 1965a, Ap. J, 141, 83 (Paper I). 1965b, ibid., 142, 925 (Paper II). 1967, ibid. (in press) (Paper III).

Faulkner, J. 1966, $A p$ J., 144, 978.

Greenstein, J. L. 1958, Encyclopedia of Physics (Berlin: Springer-Verlag), 50, 161.

Griem, H. R. 1964, Plasma Spectroscopy (New York: McGraw-Hill Book Co.), pp. 93-95.

Hamada, T., and Salpeter, E. E. 1961, Ap. J., 134, 683.

Matsushima, S. 1966 (preprint).

Pavlovskaya, E. D. 1956, Astr Zh. 33, 660.

Popper, D. M. 1954, Ap. J., 120, 316.

Terashita, Y., and Matsushima, S. 1955, Ap. J. Suppl, 13, 461.

Uesugi, A. 1962, Contr. Inst. Ap. Kyoto, No. 117.

Weidemann, V. $1960, A p . J ., 131,638$. . 1963, Zs. f. Ap., 57, 87.

1966, J. Quant. Spectrosc. Rad. Transf , 6, 691.

Copyright 1967 The University of Chicago Printed in U S A. 